Rhode Island College

Digital Commons @ RIC

\title{
Nurse Telephone Follow-up Post Initial Chemotherapy: A Quality Improvement Project
}

Katie Cherenzia

Rhode Island College

Follow this and additional works at: https://digitalcommons.ric.edu/etd

Part of the Nursing Commons

\section{Recommended Citation}

Cherenzia, Katie, "Nurse Telephone Follow-up Post Initial Chemotherapy: A Quality Improvement Project" (2017). Master's Theses, Dissertations, Graduate Research and Major Papers Overview. 187.

https://digitalcommons.ric.edu/etd/187

This Major Paper is brought to you for free and open access by the Master's Theses, Dissertations, Graduate Research and Major Papers at Digital Commons @ RIC. It has been accepted for inclusion in Master's Theses, Dissertations, Graduate Research and Major Papers Overview by an authorized administrator of Digital Commons @ RIC. For more information, please contact digitalcommons@ric.edu. 
NURSE TELEPHONE FOLLOW-UP POST INITIAL CHEMOTHERAPY: A QUALITY IMPROVEMENT PROJECT

by

Katie Cherenzia

A Major Paper Submitted in Partial Fulfillment

of the Requirements for the Degree of

Master of Science in Nursing

in

The School of Nursing

Rhode Island College

2017 


\begin{abstract}
With the evolution of cancer care and chemotherapy agents over the last 15 years there has been an evident shift in care from the inpatient to the ambulatory setting. There is a growing need for cancer care, particularly in the ambulatory (outpatient) setting. Patients receiving chemotherapy in the outpatient setting return home immediately after completing treatment and do not have direct medical and nursing supervision to monitor for and address side effects or adverse reactions of treatment in a hospital inpatient setting. Nurse telephone follow-up post initial chemotherapy allows for assessment and timely management of potential side effects experienced after the administration of chemotherapy in the outpatient setting. The purpose of this quality improvement project was to develop a standardized nurse telephone follow-up procedure post initial chemotherapy. The author developed an electronic script guideline and documentation tool along with a process for conducting the nurse-initiated calls. The project design included an educational activity and a post evaluation of the telephone follow-up procedure. Fifteen biotherapy/chemotherapy nurses participated in a four week pilot. During the four week pilot period 14 out of 34 first time chemotherapy patients received telephone follow-up utilizing the script guidelines. Ten nurses $(\mathrm{N}=10,67 \%)$ completed the post evaluation survey. Results indicated that overall the script guidelines for telephone follow-up post initial chemotherapy was well received by staff and utilized appropriately. Replication of this project should include examining the impact of telephone follow-up on patient satisfaction and outcomes. The APRN is essential in promoting evidence-based practice and bringing it into the daily practices of staff nurses to improve the quality of care for patients and their families.
\end{abstract}




\section{Table of Contents}

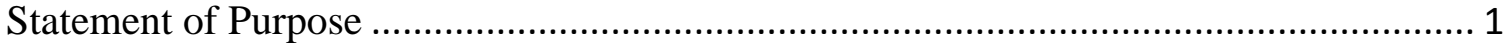

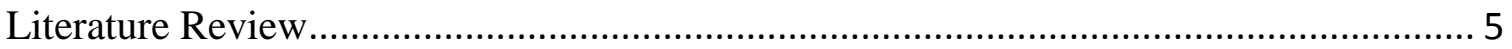

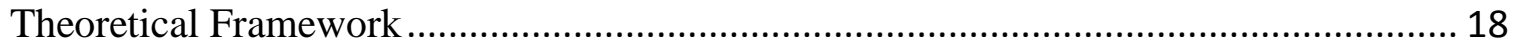

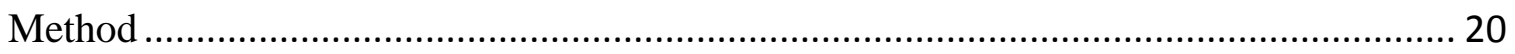

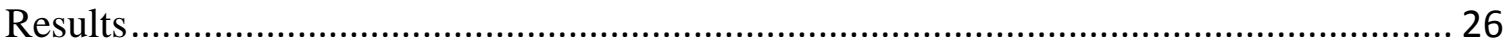

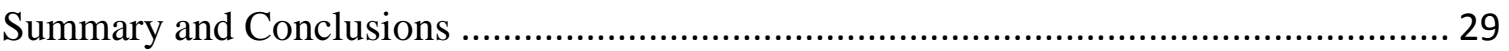

Recommendations and Implications for Advanced Nursing Practice ............................ 33

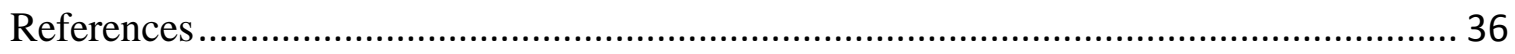

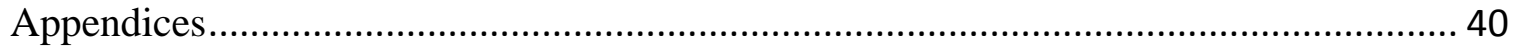




\section{NURSE TELEPHONE FOLLOW-UP POST INITIAL CHEMOTHERAPY: A QUALITY IMPROVEMENT PROJECT}

\section{Background/Statement of the Problem}

In the constantly changing world of cancer care, it is important to recognize the shift in care from inpatient to the ambulatory setting as part of the national progression within healthcare delivery. There is also increased demand for cancer care, influenced in part by the increase in newly insured patients. Millions of uninsured signed up for health insurance last year because of the Affordable Care Act insurance mandate. Included in the pool of newly insured people are cancer patients and survivors who had previously struggled to find adequate, affordable coverage because of their history of cancer. Also contributing to the demand for cancer care is the burden of the aging population, associated with increased number of cancer cases and cancer remaining the leading cause of death (American Society of Clinical Oncology, 2015). Finally, the cancer survival rate for five years is increasing, and so then are the number of cancer survivors increasing demand for cancer care (National Cancer Institute, 2015). The growing number of cancer survivors will require long-term follow-up and monitoring to detect and treat recurrence or new cancers and manage long-term treatment side effects.

The American Cancer Society (2015) estimates that 1,658,370 new cases of cancer will be diagnosed in 2015, which is down approximately 7,000 cases from 2014. Despite the decrease in the number of new cases, there is a growing population of first time oncology patients receiving initial chemotherapy in the outpatient setting (American Society of Clinical Oncology), magnifying the need for improved nurse to patient communication after first (initial) chemotherapy treatment. Patients receiving 
chemotherapy in the outpatient clinic setting return home immediately after completing treatment and do not have direct medical and nursing supervision to monitor for and address side effects or adverse reactions of treatment. Patients may even return home with chemotherapy infusing for 48-72 hours with a home infusion pump. The initiation of chemotherapy is an extremely anxiety provoking experience for many patients and their families, whether inpatient or outpatient. Patients' primary concern is what to expect during and immediately following treatment. Patients are at an increased risk of developing and experiencing symptoms while at home that require medical care (emergency department or admission). These symptoms can include pain, dyspnea, nausea, vomiting, diarrhea, constipation and mucositis (Mooney, 2002). Patients experiencing the side effects of treatment at home, as opposed to inpatient, need adequate resources and guidance to avoid hospital admissions or visits to the emergency department (Mooney). In addition to the acute symptoms patients face, long-term problems accompany cancer treatment such as depression, anxiety, fear of recurrence, loneliness, body image changes, and effects on family relationships, social environment and finances (Mooney). Chemotherapy-related symptoms disrupt normal daily living activities and diminish quality of life (Hassett, 2011). Open communication between the nurse and patient will contribute to coordinated and comprehensive patient-centered care.

Chemotherapy administration in the ambulatory setting is seen as economical and preferable for most patients (Mooney, 2002). The United States Food and Drug Administration (FDA) approved 18 new cancer treatment drugs and biologics in 2013 to bring the total number of FDA-approved anticancer drugs to more than 170 (American Society of Clinical Oncology, 2015). The availability of safer and better treatments, 
along with an expanded focus on value and quality of cancer care, has contributed to increasing survival rates and improved quality of life for patients living with cancer (American Society of Clinical Oncology, 2015). Targeted therapies work at the molecular level and are tailored to each cancer, leading to the ability to target serious and life threatening conditions (Gerber, 2008).

The most frequent reason a patient calls a cancer clinic is to report symptoms or clarify questions about plan of care or treatment (Flannery, 2009). After returning home from the clinic, many patients and family members find themselves struggling to remember all the information reviewed throughout the treatment process along with symptoms to monitor. Many patients are unable to adequately manage side effects at home and do not seek effective follow-up care. Cancer patients receiving chemotherapy require accurate and reliable information in order to adequately perform self-care and cope with the side effects of treatment (Flannery). Ensuring that the patient or their family is contacted 24-48 hours after initiation of chemotherapy may alleviate anxiety, knowledge deficit and provide prompt follow-up of symptoms and concerns along with reinforcement of the care plan.

Post chemotherapy telephone follow up by the nurse allows for open communication and provides reassurance to the patient related to their cancer care (Cox, 2003). A primary benefit of telephone follow-up is the ability to assess patients in a timely manner for potential complications that require further evaluation or hospital admission (Lynch, 2010). The nursing assessment and collaboration with MD will allow for timely management of symptoms and the potential to decrease hospital admission or emergency department visits. Along with increased communication, concise 
documentation of nurse communication post initial chemotherapy is essential to providing quality cancer care (Lynch, 2010). Documentation in the patient record allows all members of the patient's care team to be aware of any interventions or education that was provided during the telephone follow-up. Continuity of care is desired in all settings, particularly in ambulatory, where there is a strong potential for fragmented care (American Society of Clinical Oncology, 2015).

Telephone follow-up can be essential to timely management of chemotherapy side effects; however it must be completed using a standardized process. Currently patients and their families are instructed to call the clinic if they have any questions or concerns following chemotherapy administration. Instructing the patient to call with any questions or concerns can lead to the potential for missed follow-up due to an increased number of incoming calls. Taking a proactive approach would decrease the number of incoming calls to the clinic and allow for prompt follow-up with the patient. In the cancer center at Rhode Island Hospital there is no protocol or process in place for nurse telephone followup after the initiation of chemotherapy. The purpose of this quality improvement project is to develop and pilot a nurse telephone follow-up procedure post initial chemotherapy.

A review of relevant literature will be presented next. 


\section{Literature Review}

A literature review was performed using the CINAHL, UpToDate, and Pub Med databases and using keywords cancer care, chemotherapy, follow-up, side effects, adverse events, and telephone follow-up. The search was limited to materials in English from 1999- present.

\section{Epidemiology of Cancer Care}

In 2014, significant progress was made in improving the quality of cancer care as demonstrated by growth in the number of new drugs and technologies approved by the Food and Drug Administration, improvements in the five-year cancer survival rates for many cancer types, and the increasing number of cancer survivors. According to the American Cancer Society, the five year survival rate increased from 55\% in 1987-1989 to $68 \%$ in 2004-2010 (2015). However, by 2030 the number of new cancer cases in the United States will increase by $45 \%$, from 1.6 million to 2.3 million cases annually (American Society of Clinical Oncology, 2015). Along with the increase in cancer cases comes a need for improved quality of cancer care. The course of disease in patients has become increasingly unpredictable, resulting in equally unpredictable adverse events, which often lead to unscheduled hospitalizations (Coriat, 2012). An integrative approach is required to address the unpredictability identified in cancer care that involves a multidisciplinary approach and consistent follow-up. The administration of ambulatory chemotherapy means that patients will not be under the care of a healthcare provider when they are most likely to experience side effects.

\section{Chemotherapy: Targeted vs. Traditional}


Chemotherapy is a systemic therapy as drugs are distributed throughout the body by the bloodstream and be limited by the toxic effects on normal tissue (Polovich, 2014). Chemotherapy works by stopping or slowing the growth of cancer cells, which grow and divide quickly (Polovich). The array of cancer treatments available continues to grow with time and further developments in the field. Traditional cytotoxic chemotherapy targets rapidly dividing cells including cancer cells and certain normal tissues (Gerber, 2008). Cytotoxic chemotherapy works primarily through the inhibition of cell division and in addition to cancer cells, other rapidly dividing cells (e.g., hair, gastrointestinal epithelium, bone marrow) are affected by these drugs (Gerber). Traditional cytotoxic chemotherapy remains the treatment of choice for many malignancies; however targeted therapies are now a component of treatment for many types of cancer.

Targeted therapies are currently the focus of much anticancer drug development. Targeted therapies are drugs or other substances that block the growth and spread of cancer by interfering with specific molecules that are involved in the growth, progression, and spread of cancer (National Cancer Institute, 2015). The use of targeted therapies grew from 11\% in 2003 to $46 \%$ in 2013 (American Society of Clinical Oncology, 2015). Targeted therapies differ from standard chemotherapy in a number of ways

-Targeted therapies act on specific molecular targets that are associated with cancer, whereas most standard chemotherapies act on all rapidly dividing normal and cancerous cells.

-Targeted therapies are deliberately chosen or designed to interact with their target, whereas many standard chemotherapies were identified because they kill cells. 
-Finally targeted therapies are often cytostatic (that is, they block tumor cell proliferation), whereas standard chemotherapy agents are cytotoxic (that is, they kill tumor cells) (National Cancer Institute, 2015).

Despite advanced in chemotherapy, all therapies have significant and potentially serious side-effects.

\section{Chemotherapy: Side Effects}

General. Symptoms that patients experience often are congruent with the type of treatment received and vary through the course of treatment. It is a continuous challenge for healthcare professionals to enhance the patient's ability to cope with side effects during treatment. Knowledge of common symptoms enables the nurse to provide the patient with a predictable symptom profile, which represents a range of normal experiences during and after cancer treatment (Pedersen, 2012). Patient information needs to be tailored according to the patient's background, prior understanding, and level of anxiety (Pedersen). It has also been identified that due to an increase in the number of outpatients and demand for efficiency, patients experience a lack of access to healthcare providers, communication difficulties and information overload (Pedersen).

Henry, Viswanathan, Elkin, Traina, Wade, and Cella (2008) conducted a crosssectional survey to examine the prevalence of chemotherapy associated side effects and related treatment burden. Identifying and treating the side effects associated with cancer treatment can reduce their negative impact on patients' daily lives and health-related quality of life. The survey conducted by phone or completed online assessed fatigue, anxiety, depression, pain, alopecia, nausea, vomiting and additional symptoms. A total of 
814 patients reported receiving chemotherapy at the time of survey and were included in the results of the survey. The majority of patients, $88 \%$, reported at least one side effect of their treatment. Fatigue (80\%), pain (48\%), nausea and/or vomiting (48\%), anxiety (46\%), and insomnia (45\%) were the most common side effects reported (Henry). The high prevalence of chemotherapy associated side effects identified in the survey underlines the need for improvement assessment and management of said side effects.

Hassett, Rao, Brozovic, Stahl, Schwartz, Maloney, and Jacobson (2011) found that gastrointestinal problems, such as nausea, vomiting, or diarrhea, were responsible for the largest percentage of chemotherapy related hospitalizations followed by infectious problems such as pneumonia or febrile neutropenia. Among the 2,068 cancer patients identified in the prospective cohort study, 826 total hospitalizations were identified between January 2003 and December 2006. Of the identified hospitalizations, 262 (26.3\%) were classified as chemotherapy related. It was found that there were five chemotherapy related hospitalizations on average per month. Two thirds of chemotherapy related hospitalizations occurred during the first two cycles of chemotherapy; only $8 \%$ occurred after the fourth cycle. Chemotherapy related side effects and toxicities must be assessed as part of routine clinical practice particularly during the beginning cycles of chemotherapy to properly manage potential adverse effects.

Targeted Chemotherapy. The proper management of targeted chemotherapy side effects can lead to decreased risk of dose reduction, dose delay, or termination of therapy (McIntyre, 2015). Patient education is essential and patients need to be alerted about what to expect during treatment including nursing education regarding the patients 
of treating side effects early, rather than waiting until they become more severe (McIntyre). Despite the high selectivity of targeted therapies, a range of previously unknown and sometimes unpredictable side effects can emerge (Widakowich, 2007). Majority of side effects are directly related to the specific molecular target in normal tissues inhibited or modulated by the specific drug. Cutaneous toxicity is seen with the inhibitors of epidermal growth factor receptor (EGFR) and the most commonly observed side effect is an acneiform rash (Widakowich). The acneiform rash is characterized by erythematous follicular papules and pustules that appear in areas rich in sebaceous glands. Usually, the acneiform rash appears a few days after the start of anti-EGFR treatment and is more intense at weeks 2 or 3 of treatment (Widakowich). Gastrointestinal toxicity with anti-EGFR therapies presents as post-chemotherapy diarrhea and can also be a dose-limiting toxicity for most small molecule EGFR (Widakowich).

Adverse Event Reporting. Adverse event reporting in oncology is inherently complex. An adverse event as defined by the National Cancer Institute (2015) is any untoward medical occurrence associated with the use of a drug, whether or not considered drug related (abnormal lab finding, symptom, or disease) The National Cancer Institute's Common Terminology Criteria for Adverse Events (CTCAE) is the standard approach for documenting adverse events in cancer clinical trials (Trotti, 2007). Common Terminology Criteria for Adverse Events is designed as an instrument to be used to document adverse events identified through a combination of clinical and laboratory evaluation. The system is also used in the clinical setting to support treatment decisions, symptom management and medication administration. Clinician capture of 
subjective data requires first patient report of the symptom then the clinician making an assessment and interpreting the symptom (Badalucco, 2011). Common Terminology Criteria for Adverse Events tools have not been through a formal development or validation process; however some content validity is conferred through 30 years of widespread use and iterative improvements (Trotti, 2007).

\section{Use of Telephone Contact for Follow-Up and Symptom Management}

Patient Calls to Oncology Clinic. Lucia, Decker, Israel, and Decker (2007) studied the number, volume, and treatment characteristics of all patient related telephone calls to a cancer nurse at a community oncology practice during a one week period. There were a total of 332 patient calls reviewed with $30 \%$ of calls pertaining to prescriptions and $20 \%$ to symptom management (Lucia et al.). Lucia et al. found that for $87 \%$ of the calls the triage nurse was able to assist the caller without any further intervention. The utilization of oncology nurses for telephone triage in the ambulatory oncology setting demonstrates the effectiveness of resolving patient issues thereby reducing the cost of care for patients related to unnecessary emergency department visits or hospitalizations (Lucia et al.).

In a descriptive retrospective study conducted by Flannery, Phillips, and Lyons (2009), the telephone calls to medical oncology and hematology were analyzed. A telephone call record instrument was used to analyze data along with a demographic and

medical data instrument. Flannery et al. identified 5, 283 telephone calls, involving 1,486 individuals, were placed or received in a four month time period. It was found that the most frequent reasons for calling were to request diagnostic or blood work results, report 
symptoms, and to clarify questions about plan of care or treatment. The top three patient populations identified by number of calls were hematology, breast cancer, and gastrointestinal cancer. The data gathered demonstrates that telephone calls are a large component of ambulatory oncology practice.

\section{Nurse-initiated Telephone Follow-up; Non-Oncology Population. Nesari,} Zakerimoghadam, Rajab, Bassampour, and Faghihzadeh (2010) examined the effect of telephone follow-up on adherence to a diabetes therapeutic regimen. Random sampling, along with screening, was utilized to obtain a sample size of 60 patients with a diagnosis of type 2 diabetes who attended the Iranian Diabetes Society. The study was conducted over a three month period using a data sheet to record hemoglobin A1C level and a selfreported questionnaire. Study participants attended a three day diabetes self-care education program where upon completion there were randomly assigned to the experimental or control group. The experimental group received telephone follow-up from a master's nursing degree student for 12 weeks, twice per week for the first month and then weekly for the second and third months. During each telephone call, health behaviors, including diet, exercise, medication-taking, foot care, and regular monitoring of blood glucose were assessed, education reinforced, and the participants questions answered. It was found that the experimental group, after 12 weeks of telephone followup, had significantly higher diet, exercise, foot care, and blood glucose monitoring adherence compared to the control group. The experimental group $(n=30)$ reported $73 \%$ adherence to diet compared to $64 \%$ in the control group. Exercise adherence was significantly higher in the experimental group, $56 \%$, versus the control group at $22 \%$. The experimental group reported $89 \%$ medication adherence while the control group 
reported $78 \%$. Nesari et al. identified that telephone follow-up by a nurse improved the level of glycemic control.

Burch (2012) reviewed the benefits of nurse-led telephone follow-up after major colorectal surgery. The aim was to ensure a safe transition from hospital to home and additionally to examine how patients recover at home. Telephone calls were made by the enhanced recovery nurse within four weeks of surgery, with an ideal timing of two weeks following discharge home from the hospital. The telephone follow-up was conducted using a standardized document that included questions on diet, mobility, pain, wound healing, and bowel and stoma function. Two hundred patients were contacted by telephone in the 10 month period following discharge home. Of the 200 patients, $75 \%$ $(n=150)$ were contacted within the first two weeks. Intervention needed as a result of making the call was only monitored for the latter 100 patients and led to difficulty in assessing whether a problem had been prevented by making the telephone call. Of the 100 patients with intervention tracked, $76 \%$ of the calls were for advice or suggestion and $27 \%$ were to arrange medical clinic follow-up. Ninety-four patients expressed 102 concerns including wounds $(8 \%)$, bowels $(11 \%)$, stoma-related topics $(5 \%)$, lack of energy (3\%), and pain (3\%). Patients found telephone follow-up suitable to meet the needs with advice giving for symptom management and providing reassurance on issues of concern.

Stolic, Mitchell, and Wollin (2010) conducted a literature review of nurse-led telephone interventions for people with cardiac disease. Inclusion criteria for literature included telephone intervention, people with cardiac disease, post discharge period, nurse delivered and published 1980-2009. Twenty-four articles met the inclusion criteria and 
were further reviewed. Of the twenty four studies reviewed, fifteen had at least one positive finding. Positive effects were reported for seven studies in all outcome measures including hospital readmission and mortality, attendance to cardiac rehabilitation program, risk factor behaviors, and quality of life (Stolic). Mixed results with both positive findings and no statistically differences detected were reported with eight studies. Positive findings for risk factors, quality of life, psychological status, selfefficacy, and resource use were reported in the mixed studies (Stolic). Nine studies reported no differences detected in any outcome measure including risk factors, attendance to cardiac rehabilitation program, quality of life, psychological status, post discharge complication, and satisfaction (Stolic). In the nine studies with no differences detected similarities such as study rigor or sub optimal design, non-expert nurse providing intervention and a fewer number of phone calls were identified. Nurse-led telephone intervention may help people with cardiac disease for a variety of outcomes including reducing risk factor behaviors, improving physical functioning, improving psychological status, reducing hospital consumption and postoperative complications (Stolic).

\section{Nurse-initiated Telephone Follow-Up: Oncology}

In a study by Kelly, Faught, and Holmes (1999), telephone follow-up was examined in the setting of ovarian cancer treatment with the objective to assess the impact and any benefit received from telephone follow-up between chemotherapy treatments. The gynecology oncology liaison nurse-initiated telephone follow-up five to seven days after the patient received treatment. The study was conducted in a one year period with 31 patients responding to the questionnaire assessing the impact of telephone follow-up 
between chemotherapy treatments. The liaison nurse would make appropriate referrals to other members of the patient's care team if assessment demonstrated further needs and guidance. A standardized assessment tool was used for documentation of the follow-up call that included gastrointestinal symptoms, activity, elimination, and neurotoxicity. By telephoning patients five to seven days after they had received chemotherapy, nurses were able to aide patients in management of symptoms, assessment of side effects, and promotion of self-care. Patients received a call-back study questionnaire to provide feedback regarding benefits and perception of care. It was found that $87 \%$ of the 31 patients viewed telephone follow-up post chemotherapy as helpful. With regard to issues/concerns being addressed during the telephone call, $80 \%$ responded that their issues had been addressed appropriately. According to the study findings, telephone follow-up post chemotherapy made a positive impact on the patient's perception of their care.

In a prospective and descriptive pilot study, Mooney, Beck, Friedman, and Farzanfar (2002) examined telephone-linked care (TLC). Telephone-linked care is a computer-based telecommunications system developed to help clinicians care for patients with chronic health conditions. The system was used to monitor symptoms of patients that had received chemotherapy. Patients would call into the system and report symptom experiences through an automated system that would then alert the healthcare team by fax of symptoms exceeding a specified threshold of severity. Patients were instructed to call the system beginning 24 hours after chemotherapy and continue daily until their next treatment cycle. A total of 27 patients were enrolled into the study. It was found that $67 \%$ $(n=27)$ of patients experienced symptoms severe enough to generate an alert, highlighting the need for improved communication methods. An adherence rate was computed for each 
participant based on the number of times the participant actually called divided by the number of anticipated calls (based on length of chemotherapy cycle) and a mean adherence rate of $75 \%$ was found. Patients often did not take the initiative to call their providers even when symptoms are of moderate or greater severity or when they are causing the patient significant distress. Only two patients reported calling their nurse or physician to ask for assistance with severe symptoms. Patients were instructed to call their provider to seek symptom control even when reporting to the TLC system; the system was not to be seen as a substitute for direct communication with provider (Mooney)

Cox and Wilson (2003) conducted a literature review on nurse led follow-up for patients who have completed treatment, with a particular focus on telephone interventions. Thirty-seven papers were reviewed relating to follow-up services for patients with cancer. Many studies examined the appropriateness and effectiveness of follow-up upon completion of cancer treatment. Surveillance could be better served by a general practitioner or a nurse in the terms of physical, social, and psychological rehabilitation (Cox). Cox et al. identified that nurse-led intervention was effective in managing symptoms along with greater satisfaction than conventional care and a decrease in costs by $31 \%$. Telephone follow-up was seen as a cost-effective method of communicating with patients as part of their follow-up care and has consistently been shown to be a feasible and effective way of improving support and symptom management post chemotherapy. Intervention by telephone allowed for early identification of problems before they progress to crises, in turn reducing the number of admissions and helping to redirect patients to the most suitable resources (Cox). 
Providing prompt recognition and treatment of toxicity related to chemotherapy is essential to optimal outcomes (Lynch, 2010). Optimal outcomes can be achieved by prevention and early detection of toxicities that lead to improved treatment adherence, decreased costs, and overall improved treatment outcomes. Telephone follow-up is an efficient means to providing a patient and their family with optimal care during treatment. In a quality improvement project conducted in an urban community cancer center in Pennsylvania addressing follow-up in the older adults, patient's side effects could be treated via phone or an urgent office visit, not hospitalization. The telephone follow-up calls were completed by the chemotherapy nurse or geriatric social worker with the purpose of improving care for the older adult receiving chemotherapy. Patients were called every other day for a total of five calls and a standard documentation tool was utilized. Of the 18 patients identified, only seven patients experienced complications during chemotherapy treatment. A total of four patients had not yet completed treatment during the study; however they did not experience any complications. Two patients, with stage III or IV disease, were admitted to the hospital for supportive care after the first chemotherapy cycle. A limitation to the quality improvement project was only 18 patients were enrolled for telephone follow-up due to inadequate resources.

In a retrospective study conducted at the Cochin Hospital Department of Oncology in France, chemotherapy-induced unscheduled hospitalizations rates were reviewed (Coriat, 2012). The study was conducted in August 2008 and included all consecutive patients receiving chemotherapy infusions with no exclusion criteria identified. A total of 259 outpatients were identified that received 2,374 cycles of chemotherapy. A standardized five-step procedure with the initial steps involving clinical evaluation, 
chemotherapy prescription, and preparation of chemotherapy prior to treatment was used for hospital-home monitoring program. The last step of the hospital-home monitoring program included the physician conducting follow-up with patient by calling him or her twice, on the second and eighth days after chemotherapy, to collect data on treatment toxicity and potentially adverse events and to provide patient with recommendations. These could range from setting up an appointment with the physician to identifying whether the patient needs hospitalization and continuing to educate the patient. It was found that telephone follow-up was a cost-effective strategy for offering ambulatory chemotherapy treatment to patients with cancer. Coriat found that there was a reduction in the frequency of unexpected hospitalizations with the use of the hospital-home monitoring program: $4.9 \%$ down to $2.4 \%$ with the program. The program allowed for patients to address questions regarding medications and chemotherapy management while the providing reinforcement of close physician follow-up.

Nurse-initiated telephone follow-up in both non-oncology and oncology populations can have a positive impact on patient outcomes and quality of life. Specifically in the oncology population, nurse-initiated telephone follow-up led to an improvement in patient's perception of care, symptom management, and unplanned hospitalizations. Telephone follow-up was identified as cost effective method for follow-up in oncology patients.

Next, the theoretical framework for this quality improvement project will be presented. 


\section{Theoretical Framework}

Lewin's Change Theory will be utilized to guide this quality improvement project. The theory examines the process of planned change. Planned change is described as occurring by design, as opposed to change that is spontaneous or that occurs by happenstance or by accident. Making a change means trying to break a wellestablished custom or social habit (Lewin, 1951). According to Lewin there are three phases that must occur if planned change is to be successful: unfreezing the status quo, moving to a new state, and refreezing the change to make it permanent (McEwen, 2011). Using this theory effective change can be implemented.

The concepts of field and force are central to Lewin's theories on planned change. Lewin states that change is a move from the status quo that results in disequilibrium between opposing forces (Lewin, 1951). A field can be viewed as a system, demonstrating when change occurs in one part of the system, the whole system must be examined. Force is defined as a directed entity that has the characteristics of direction, focus, and strength.

Driving forces and restraining forces are identified by Lewin as the two forces involved in change. A driving force encourages or facilitates movement to a new direction or goal, while a restraining force has the opposite effect. Driving forces identified in this project include the support of administration, education session, and literature supporting the importance of telephone follow-up post initial chemotherapy. Restraining forces block or impede progress toward the goal. If driving forces and restraining forces can be identified, it may be possible to predict if and when change would be successful (Lewin, 1951). Some potential restraining forces identified for the 
project are reluctance of nurses to participate, adequate time to conduct telephone followup, and lack of patient/family participation when called.

During the unfreezing stage participants must be informed of the need for change and should agree to change is needed. The educational session conducted with staff will provide the opportunity to highlight the need for a change in practice and encourage staff buy-in. The process of unfreezing involves finding a method of making it possible for people to let go of an old pattern or habit that was counterproductive in some way (Lewin, 1951). During the unfreezing process staff will be presented with the process for telephone follow-up in the clinic and the template for documentation. Change in the work environment can lead to feelings of uneasiness, uncertainty, and loss of control (McEwen, 2011). Driving forces should exceed restraining forces during the second phase, moving to a new state, of the planned change process. The refreezing phase is when stabilization occurs and if successful change is assimilated into the system. Permanency of new level, or permanency for a desired period, should be included in the objective (Lewin, 1951). Permanency implies that the new force field is made relatively secure against change. The pilot process will be viewed as moving to a new state of practice and if implementation is agreed upon the protocol will be reviewed with all staff as part of daily practice.

Next, study methods will be presented. 


\section{Method}

\section{Purpose}

The purpose of this quality improvement project was to develop a nurse telephone follow-up procedure post initial chemotherapy.

\section{Design}

The project design included an educational activity and a post evaluation of the telephone follow-up procedure.

\section{Site and Sample}

Target sample was fifteen biotherapy/chemotherapy certified nurses in the Comprehensive Cancer Center at Rhode Island Hospital, an ambulatory clinic with an infusion area consisting of 26 treatment chairs/stretchers.

\section{Procedures}

Brief background. Prior to the pilot period, nurses attempted to make post initial chemotherapy follow-up calls as an expectation of their role; however there was not a standardized process or tool for documentation of the follow-up call. Therefore calls were not always being conducted, in part, due to lack of standardization and time. The process was that follow-up calls were documented in a narrative progress note. Assessments varied from nurse to nurse conducting the call. Patients and families were instructed to call the clinic with any questions or concerns post initial chemotherapy treatment. They were not informed that a nurse would contact them by phone in about 48 hours after treatment. Given lack of standardization and documentation of the process, 
nursing had not fully embraced telephone follow-up despite recognition of the need for timely follow-up calls for oncology patients.

Previous to this project, the researcher developed a standardized documentation tool in October of 2015 with assistance and approval from the leadership team at the cancer center. The tool was created utilizing a standard assessment template for conversion into a documentation tool in the EHR for the pilot period. The assessment tool (Appendix A) was developed using common themes identified in research including assessment of gastrointestinal issues (nausea, vomiting, constipation, and diarrhea), activity level/fatigue, and risk for infection, medication review, and clinic follow-up. The symptoms most experienced post chemotherapy were graded utilizing the Common Terminology Criteria for Adverse Events (CTCAE) from the National Institutes of Health and National Cancer Institute (2010). Grading of symptoms/adverse events refers to the severity of the adverse event and the CTCAE displays grades 1 (minimal or no symptoms) through grade 5 (severe symptoms) with unique clinical descriptions of severity for each adverse event. The chemotherapy follow-up assessment documentation tool was individualized and customized within the system to have patient specific information automatically populate the documentation such as treatment plan and attending physician. There was little research to suggest how long the follow-up call would last, but based on the number of questions and allowing time for patient discussion, it was estimated each call would require approximately 15 minutes.

The documentation tool was built into the EHR in August of 2016 by the researcher with the assistance of the unit based Quality and Safety Manager. The student 
researcher conducted implementation of the standardized tool, nurse education and the pilot in September of 2016.

Proposed change. This quality improvement project piloted a new standardized tool and procedure for nurse-initiated telephone follow-up with patients post initial chemotherapy treatment. A post initial chemotherapy follow-up script with assessment tool for adverse events was piloted (Appendix A). The telephone assessment utilized the researcher-developed, customized chemotherapy follow-up script with the documentation template described previously. The nurse-initiated follow-up phone call script was added for the purpose of this quality improvement project.

\section{Procedures}

Permission for the pilot was obtained from the Director of the Comprehensive Cancer Center as well as the clinical manager for the Comprehensive Cancer Center at Rhode Island Hospital. Prior to beginning the pilot, IRB determination of this proposal as a quality improvement initiative was made by Lifespan IRB and Rhode Island College IRB.

When a patient was in the clinic receiving their first chemotherapy treatment, the nurse would notify the charge nurse of the first time patient. Typically, the nurse caring for the patient on the first day of chemotherapy would remain the patient's primary nurse throughout their treatment course. At the time of the first chemotherapy treatment, the patient was informed that a follow-up call would be made in about 48 hours. The patient could designate a family member or friend to discuss their care with the nurse when the call was conducted. The nurse would document the telephone encounter within the 
customized chemotherapy follow-up assessment screen in the notes section of the EHR.

The nurse would also document any indicated follow-up plans in the assessment screen.

After the documentation/assessment tool was developed and built within the EHR, the participating nurses attended a one-hour education session. The purpose of the education session was to review the researcher developed assessment tool and process for utilization within the EHR. Importance of telephone follow-up and standardization of the process were also explained during the session. Table 1 outlines the content of the education session.

Table 1

Education Session Outline

\begin{tabular}{|c|c|c|}
\hline Objectives & Content & $\begin{array}{l}\text { Process For Conducting } \\
\text { Call and Documentation }\end{array}$ \\
\hline $\begin{array}{l}\text {-Review development and } \\
\text { implementation of } \\
\text { standardized } \\
\text { documentation/assessment } \\
\text { tool }\end{array}$ & -Purpose of project & $\begin{array}{l}\text {-Call to be conducted } \\
\text { within } 48 \text { hours to all first } \\
\text { time chemotherapy patients }\end{array}$ \\
\hline $\begin{array}{l}\text {-Identify the driving force } \\
\text { for telephone follow-up } \\
\text { change }\end{array}$ & -Development of tool & $\begin{array}{l}\text {-Documentation under } \\
\text { telephone encounter within } \\
\text { EHR with follow-up } \\
\text { (MD/NP/PA) initiated for } \\
\text { CTCAE score }>3\end{array}$ \\
\hline $\begin{array}{l}\text {-Identify process for } \\
\text { identification of } \\
\text { appropriate patients and } \\
\text { assignment of staff }\end{array}$ & $\begin{array}{l}\text {-Review of current } \\
\text { literature }\end{array}$ & -Support of management \\
\hline $\begin{array}{l}\text {-Identify and demonstrate } \\
\text { competency in the } \\
\text { elements of assessment in }\end{array}$ & -Review of current process & $\begin{array}{l}\text {-Utilization of charge nurse } \\
\text { to allow for time for call } \\
\text { backs }\end{array}$ \\
\hline
\end{tabular}




\begin{tabular}{|l|l|l|}
\hline $\begin{array}{l}\text { the post initial } \\
\text { chemotherapy call } \\
\text { guidelines }\end{array}$ & $\begin{array}{l}\text {-Discussion of potential } \\
\text { barriers }\end{array}$ & $\begin{array}{l}\text {-Competency with } \\
\text { documentation of call } \\
\text { guidelines }\end{array}$ \\
\hline
\end{tabular}

The majority of the education session was dedicated to the background of the quality improvement project to provide the nurses with an understanding of the development of the tool along with the need for change. The importance of follow-up with provider or LIP for any CTCAE score $\geq 3$ or uncontrolled symptoms was also stressed during the session. Provider follow-up or intervention was identified as necessary for a CTCAE score $\geq 3$ as there can be a negative impact on self-care ADLs and on maintaining adequate nutritional status, leaving the patient at risk for poor symptom management. Poor symptom management or uncontrolled symptoms in the past would lead to ED visits or hospital admissions. Competency with call guideline and assessment was demonstrated by having the nurse complete documentation on a test patient scenario within the EHR test environment during the educational session. There was time at the end of the educational session for participant questions or concerns.

Once all nurses completed the educational session, telephone follow-up calls were piloted for one month in the Comprehensive Cancer Center at Rhode Island Hospital. Nurses would identify all first time patients and assign a nurse to conduct the telephone follow-up phone call. During the pilot period it was the responsibility of the charge nurse to provide protected time for the staff nurse to conduct the telephone follow-up. Nurses would attempt to contact the patients two times within 48 hours post initial 
chemotherapy. The expected number of patients who would receive first time chemotherapy during the pilot was 15-20.

Nursing leadership and the Quality and Safety Manager for the clinic continued to reinforce the value of follow-up calls post initial chemotherapy treatment during monthly staff meetings and daily infusion room huddle.

\section{Measurement}

After the pilot period was complete, participating nurses attended a follow-up session to complete a post evaluation of the chemotherapy follow-up guidelines script (Appendix C). The evaluation looked at the ease of the tool, flow and appropriate assessment of patients' symptoms. A 5-point Likert scale was utilized on the post evaluation assessment. The post evaluation completed by staff was reviewed for validation of the chemotherapy follow-up assessment tool.

Next, results will be presented 


\section{Results}

Staff education was completed and was attended by all 15 staff nurses (100\%). The pilot period began on September 26, 2016 and ended on October 21, 2016. During the four-week pilot period a total of 34 patients received first time chemotherapy. Fourteen patients out of the $34(41 \%)$ received a telephone follow-up call within fortyeight hours of receiving first time chemotherapy.

After the pilot period was completed, 10 nurses completed the post evaluation survey (67\%). Table 2 illustrates the results of the evaluation.

\section{Table 2}

\section{Post Evaluation Results}

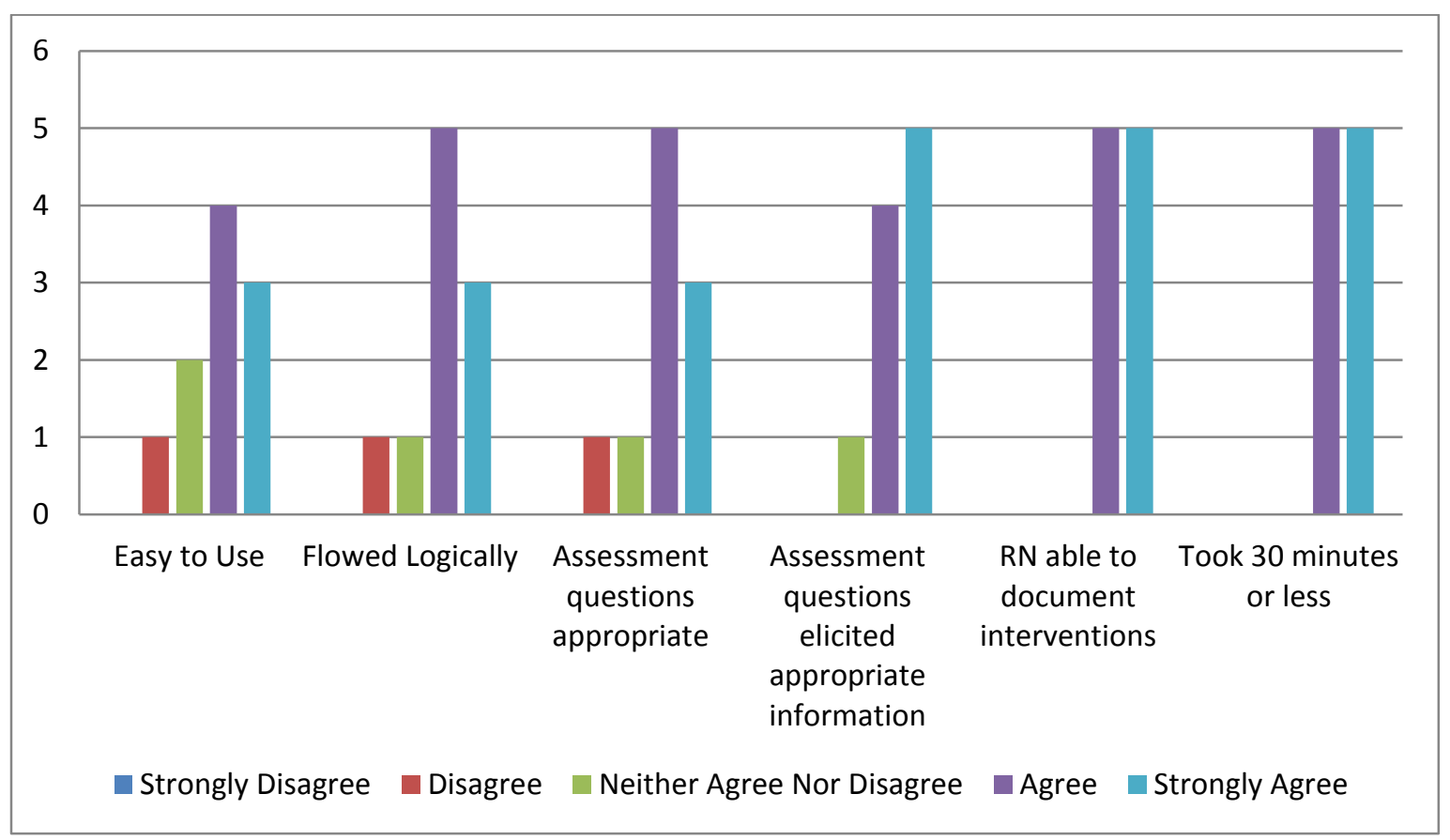

Review of the post evaluation results demonstrated that overall $70 \%$ of nurses $(n=7)$ responded strongly agree or agree for the ease of utilizing the script guideline. 
Twenty percent of the staff $(n=2)$ selected neither agree nor disagree with comments that included: "pain script difficult to complete"; "it would be more useful to have defined targets versus defined script because the conversation is often facilitated by how the patient responds to "how have you been feeling", and "difficult to access".

The majority of the staff, $80 \%(\mathrm{n}=8)$ agreed that the script flowed logically with $10 \%(\mathrm{n}=1)$ disagreeing and $10 \%(\mathrm{n}=1)$ neither agreed nor disagreed. Staff also felt strongly that the assessment questions were appropriate to post chemotherapy patients, with $80 \%$ validating the questions with strongly agree or agree. Ten percent of the staff did not feel that the questions were appropriate and included the following comment: "the time frame in which a patient gets called should be regimen specific".

Staff felt strongly when questioned regarding the assessment questions eliciting appropriate information from patient/family; $50 \%(n=5)$ strongly agreed, $40 \%(n=4)$ agreed, and $10 \%(\mathrm{n}=1)$ neither agreed nor disagreed. The staff also validated that there was adequate room for documentation of interventions performed with $50 \%(n=5)$ strongly agreed and 50\% (n=5) agreed. Finally staff evaluated the time to conduct the call and the completion of documentation with all staff agreeing the total time spent being 30 minutes or less; $50 \%(n=5)$ strongly agreed and $50 \%(n=5)$ agreed.

Additional comments on the post evaluation included: "The script would automatically open at the bottom instead of the top of the page", "Dedicated time to complete calls would have been helpful" and "Patient demographics were not being automatically pulled into the note template". 
The student researchers in collaboration with other cancer center leadership, will review all feedback and look at adjustments that can be made to further improve the template.

Next, summary and conclusions will be discussed. 


\section{Summary and Conclusions}

Patients receiving chemotherapy in the outpatient clinic setting return home immediately after completing treatment and do not have direct inpatient medical and nursing supervision to monitor for and address side effects or adverse reactions of treatment. The initiation of chemotherapy is an extremely anxiety provoking experience for many patients and their families, whether inpatient or outpatient. Patients' primary concern is what to expect during and immediately following treatment. Patients are at an increased risk of developing and experiencing symptoms while at home that require medical care (emergency department or admission). As demonstrated in the literature review, nurse-initiated telephone follow-up in both non-oncology and oncology populations can have a positive impact on patient outcomes and quality of life. Specifically in the oncology population, nurse-initiated telephone follow-up led to an improvement in patient's perception of care, symptom management and unplanned hospitalizations.

Standardization of telephone follow-up after initial chemotherapy was identified as a best practice standard to improve patient care and satisfaction as chemotherapy, increasingly is delivered in the outpatient setting. Prior to this quality improvement project, there was an expectation that the nurse would give the patient contact information to call if needed and the conversation would be documented in a progress note. There was not a standardized process or tool for documentation of follow-up post initial chemotherapy with documentation that varied from nurse to nurse. A standardized telephone follow-up tool with guidelines was developed utilizing current assessment 
CTCAE criteria. The documentation/assessment tool was implemented and piloted for one month with a post evaluation of the assessment tool.

Nurse education in the use of the on-line post initial chemotherapy assessment script occurred at a monthly nursing meeting and was attended by 12 of the 15 nurses. The remaining three nurses were educated two days later on the unit. Ten nurses out of a potential 15 nurses in the project center $(67 \%)$ completed the post evaluation questionnaire at the end of the pilot month. Due to the project design and anonymity of nurse response on the post evaluation questionnaire, it was not possible to determined why the remaining five post evaluations were not completed.

Overall the template was well received by staff and utilized appropriately for telephone follow-up. The feedback related to different chemotherapy regimens was expected and will always be a challenge when looking to standardize a process in cancer care. The script was not meant to be all inclusive of patients' signs and symptoms; however, it was designed to assess non-drug specific side effects experienced during chemotherapy. There are numerous different chemotherapy regimens for each cancer type and it would be challenging to individualize telephone follow-up specific to each regimen. Nurse will need to use their specialized knowledge base of chemotherapy protocols to tailor the use of the standard script when appropriate. The negative feedback from staff was primarily related to the functionality and limitations of the EHR. The template within the EHR had limitations such as opening automatically at the bottom of the script which cannot be addressed except for educating to this fact in the future. 
Out of the 14 calls conducted, all were completed within the 48 hour time frame with the patient or a family member. Anecdotally, patients and families responded positively to the telephone follow-up process. Staff nurses participating in the pilot commented to this writer that patients expressed appreciation for the nurse-initiated contact even if they were not experiencing side effects at that time.

Limitations were also evident. Increased volume in the clinic over the course of the pilot period posed a significant challenge in terms of protected time to complete the calls. Due to computer limitations, the schedule was not able to be blocked for protected time to conduct the follow-up. The responsibility of providing protected time away from patient care to conduct the follow-up calls fell to the charge nurse. Scheduling limitations of the EHR did not allow the nurse scheduled to be blocked in a formalized way to allow for protected time to the conduct the follow-up call. Also staff LOAs and vacant positions in addition to the increased volume posed a challenge to the pilot. It was predicted during the pilot period 15-20 patients would receive first time chemotherapy and the actual number of first time chemotherapy patients was 34 . Unfortunately, the staff were not able to conduct calls to all first time patients during the pilot period and may have accounted for only 10 post evaluation questionnaires being completed. The staff were responsible for determining which team member would be conducting the call based on scheduling and not every nurse was able to conduct a telephone follow-up during the pilot period. Staffing engagement was also a limitation; given the increased census, the staff was focused on the demands of increased patient volume and not necessarily engaged in conducting the telephone follow-up. 
Future replication of this quality improvement project would require protected time for staff to conduct the telephone follow-up. It must be determined how to block the schedule within the EHR, or by other means, to allow for protected time. Protected time to conduct the call would allow for an increase in the number of calls conducted and also potentially increase staff engagement. Replication of the telephone follow-up could be targeted to a specific disease group to allow for increased support for the nursing staff in their efforts to conduct the calls and utilize the tool.

In conclusion, this project demonstrated positive feedback from staff nurses, with $70 \%$ or greater strongly agreeing or agreeing that the chemotherapy follow-up assessment tool was easy to use and included appropriate of assessment questions.

Next, recommendations and implications for advanced nursing practice will be discussed. 


\section{Recommendations and Implications for Advanced Nursing Practice}

This project validated the need for a standardized process and assessment tool for nurse-initiated telephone follow-up after initial chemotherapy treatment in an outpatient cancer center. Results demonstrated staff agreed that the standardized tool asked appropriate questions and solicited appropriate answers from patients or family. The advanced practice nurse (APRN), specifically the Clinical Nurse Specialist (CNS), is in an exceptional position to identify, promote, and facilitate standardization of nurse-driven programs.

In this quality improvement project, the APRN student had the opportunity to develop a post initial chemotherapy telephone follow-up assessment tool and provide an educational opportunity for staff members. The APRN student was able to work closely, at an intradisciplinary level, with the nursing leadership of the cancer center and will continue to be involved to update the telephone follow-up assessment tool and future implementation in all cancer center sites. Prior to implementing the script at other sites, staffing concerns must be addressed including protected time to conduct the follow-up call. The leadership group will continue to refine the process in order to obtain the best outcomes for both patients and staff while moving towards embracing the oncology medical home care delivery model. The oncology medical home model is a patientfocused system of delivering quality cancer care that is efficient and coordinated.

Next steps include revising the assessment template based on feedback and collaborating with the Quality and Safety Manager to determine a process for the tracking of chemotherapy patients. Specifically, the percentage of first time patients that received 
telephone follow-up and the impact on emergency room visits and hospital admissions over time should be assessed. Patient satisfaction will also be reviewed for the potential positive impact of communication from the healthcare team, management of symptoms, and explanation of care.

The APRN is a key member of any unit-based project with impact at the level of the patient, nurse, and system as illustrated in this quality improvement project. The ability of the APRN to be involved in all three spheres of nursing influence demonstrated impact on patient care for the greater good including patient management and patient satisfaction. The telephone follow-up assessment tool addressed the patient needs while also providing patient education related to symptom management and early identification of possible complications. Ultimately, if fully implemented, the standardized post initial chemotherapy assessment could potentially have a positive impact on patient satisfaction and provide an enhanced continuum of care in the outpatient setting. Impact on nursing included nurse education and symptom management, with the ultimate goal of decreased emergency room visits and avoidable hospitalizations. The tool facilitates the nurse to promptly assess the patient for any complications and advocate on behalf of the patient for appropriate follow-up care.

The APRN is in the position to influence patient care at the policy level as well. Standardization of nurse-initiated telephone follow-up could lead to positive outcomes and also best practice guidelines in cancer care. The APRN can collaborate with cancer center leadership system wide to develop a standard of care procedure for telephone follow-up post initial chemotherapy. It will be both essential and important for the APRN to be a key stakeholder in the process of developing standardized policy/procedure 
related nurse-initiated telephone follow-up and also the maintenance of such policy/procedure to determine the impact on delivery quality cancer care. Focusing on the cancer center's quality goals for 2017 including decreased emergency room admissions, patients' willingness to recommend, and pain management satisfaction will aid in validation of the tool's impact on providing quality cancer care and alignment with the oncology medical home model.

A number of cited studies have demonstrated that nurse-initiated telephone follow-up can have a positive impact on patient outcomes; however, further research is needed. On a systems level, use of the tool and standardized process can be examined in relation to emergency room visits and admissions to the inpatient unit. The overarching goal for any cancer institution is to keep their patients out of the emergency room and their care managed as well in the outpatient setting as is feasibly possible. There is a need for further research examining the impact of telephone follow-up within the oncology medical home model. Also, future research could include standardization and validation of a tool to conduct telephone follow-up. Further research is required to evaluate nurse-initiated follow-up calls, post initial chemotherapy as a best practice.

The APRN is a key stakeholder in promoting evidence-based practice and bringing it into the daily practice of staff nurses to improve the quality of patient care provided. Future goals of this project are to examine the impact of telephone follow-up on patient satisfaction and outcomes, embodying the philosophy of the oncology medical home. Research regarding the process of a standardized tool for telephone follow-up has the potential to impact many and increase positive outcomes for chemotherapy patients. 


\section{References}

American Cancer Society: Cancer facts and figures 2015. Atlanta, GA, American Cancer Society, 2015

American Society of Clinical Oncology. (2015) The State of Cancer Care in America, 2015: A Report by the American Society of Clinical Oncology. Journal of Oncology Practice, 1-68.

Badalucco, S., \& Reed, K.K. (2011). Supporting Quality and Patient Safety in Cancer Clinical Trials. Clinical Journal of Oncology Nursing, 15(3), 263-266.

Burch, Jennie (2012). Enhanced recovery and nurse-led telephone follow-up post surgery. British Journal of Nursing, 21(16), 524-529.

Coriat, R., Boudou-Rouquette, P., Durand, J.P., Forgeot d'Arc, P., Martin, I., Mir, O., Ropert, S., Alexandre, J., \& Goldwasser, F. (2012). Cost Effectiveness of Integrated Medicine in Patients with Cancer Receiving Anticancer Chemotherapy. Journal of Oncology Practice, 8(4), 205-210.

Cox, K., \& Wilson, E. (2003). Follow-up for people with cancer: nurse-led services and telephone interventions. Journal of Advanced Nursing, 43(1), 51-61.

Flannery, M., Phillips, S.M., \& Lyons, C.A. (2009). Examining Telephone Calls in Ambulatory Oncology. Journal of Oncology Practice, 5(2), 57-60.

Gerber, D.E. (2008). Targeted Therapies: A New Generation of Cancer Treatments. American Family Physician, 77(3), 311-319. 
Hasseet, M.J., Rao, S.R., Brozovic, S., Stahl, J.E., Schwartz, J.H., Maloney, B., Jacobson, J.O. (2001). Chemotherapy-Related Hospitalization Among Community Cancer Center Patients. The Oncologist

Henry, D.H., Viswanathan, H.N., Elkin, E.P., Traina, S., Wade, S., Cella, D. (2008). Symptoms and treatment burden associated with cancer treatment: results from cross-sectional national survey in the U.S. Support Care Cancer, 16, 791-801.

Kelly, D.F. Faught, W.J., Holmes, L.A. (1999). Ovarian cancer treatment: The benefit of patient telephone follow-up post-chemotherapy. Journal of Canadian Oncology Nursing, 9, 175-178.

Lewin, K. (1951). Field theory in social science. New York: Harper and Brothers.

Lucia, V.C., Decker, V.B., Israel, C.E., Decker, D.A. (2007). Telephone contacts between triage nurses and cancer patients: an integral part of a community oncology practice. Community Oncology, 350-354.

Lynch., M.P., Marcone, D., \& King, J. (2010). Chemotherapy Follow-Up in Older Adults: Results of Quality Improvement Project. Clinical Journal of Oncology Nursing, 14(5), 619-625.

McEwen, M., Wills, E.M., (2011). Theoretical Basis for Nursing. Philadelphia, PA: Lippincott Williams \& Wilkins.

McIntyre, K. (2015). An Oncology Nurses' Guide to New Targeted Agents for Metastatic Colorectal Cancer. Clinical Journal of Oncology Nursing, 19(5), 571-579. 
Mooney, K.H., Beck, S.L., Friedman, R.H., Farzanfar, R. (2002). Telephone-Linked Care for Cancer Symptom Monitoring A Pilot Study. Cancer Practice, 10(3), 147-154.

National Cancer Institute. (2015) Cancer Statistics

National Institute of Health. (2010) Common Terminology Criteria for Adverse Events

Nesari, M., Zakerimoghadam, M., Rajab, A., Bassampou, S., \& Faghihzadeh, S. (2010). Effect of telephone follow-up on adherence to a diabetes therapeutic regimen. Japan Journal of Nursing Science, 7, 121-128.

Pedersen, B., Koktved, D.P., Nielsen, L.L. (2012). Living with side effects from cancer treatment - a challenge to target information. Scandinavian Journal of Caring Sciences, 27, 715-723.

Polovich, M., Whiteford, J.M., Olsen, M., (2014). Chemotherapy and Biotherapy Guidelines and Recommendations for Pracitce. Pittsburgh, PA: Oncology Nursing Society.

Stolic, S., Mitchell, M., Wollin, J. (2010). Nurse-led telephone interventions for people with cardiac disease: A review of the research literature. European Journal of Cardiovascular Nursing, 9, 203-217.

Trotti, A., Colevas, A.D., Setser, A., \& Basch, E. (2007). Patient-Reported Outcomes and the Evolution of Adverse Event Reporting in Oncology. Journal of Clinical Oncology, 25(32), 5121-5127. 
Widakowich, C., De Castro, G., De Azambuja, E., Dinh, P., \& Awada, A. (2007).

Review: Side Effects of Approved Molecular Targeted Therapies in Solid Cancers. The Oncologist, 12, 1443-1455. 


\section{Appendix A}

Comprehensive Cancer Center: Post Initial Chemotherapy Telephone Follow-Up Assessment

\begin{tabular}{|c|c|c|c|c|c|}
\hline \multicolumn{6}{|c|}{ Comprehensive Cancer Center: Post Initial Chemotherapy Telephone Follow-Up } \\
\hline \multirow{3}{*}{ Script } & \multicolumn{5}{|c|}{ Assessment/Narrative } \\
\hline & \multicolumn{5}{|c|}{ Patient Active Problem List } \\
\hline & $\begin{array}{l}\text { Treat } \\
\text { Date } \\
\text { Atten }\end{array}$ & $\begin{array}{l}\text { ment Plan: } \\
\text { First Chemotl } \\
\text { lding: }\end{array}$ & herapy: & & \\
\hline \multicolumn{6}{|l|}{$\begin{array}{l}\text { "How have you been feeling } \\
\text { since you received your } \\
\text { chemotherapy?" }\end{array}$} \\
\hline Pain & \multicolumn{5}{|c|}{$\begin{array}{l}\text { Pain assessment } \\
\text { Pain (activities that relieve): } \\
\text { Pain (aggravating factors): }\end{array}$} \\
\hline Infection & \multicolumn{5}{|l|}{$\begin{array}{l}\text { Fever } \\
\text { Chills }\end{array}$} \\
\hline & \multicolumn{5}{|c|}{ CTCAE V4 Grading } \\
\hline & None & 1 & 2 & 3 & 4 \\
\hline Constipation & $\square$ & \begin{tabular}{|l|} 
Occasional \\
or \\
intermittent \\
symptoms; \\
occasional \\
use of stool \\
softeners, \\
laxatives, \\
dietary \\
modification \\
or enema \\
\end{tabular} & $\begin{array}{l}\text { Persistent } \\
\text { symptoms } \\
\text { with regular } \\
\text { use of } \\
\text { laxatives or } \\
\text { enemas; } \\
\text { limiting } \\
\text { instrumental } \\
\text { ADL }\end{array}$ & $\begin{array}{l}\square \text { Obstipation } \\
\text { with manual } \\
\text { evacuation } \\
\text { indicted; } \\
\text { limiting self } \\
\text { care ADL }\end{array}$ & \begin{tabular}{|l|} 
Life- \\
threatening \\
consequences; \\
urgent \\
intervention \\
indicated
\end{tabular} \\
\hline Diarrhea & $\square$ & $\begin{array}{l}\square \text { Increase } \\
\text { of <4 stools } \\
\text { per day over } \\
\text { baseline, } \\
\text { mild } \\
\text { increase in } \\
\text { ostomy } \\
\text { output }\end{array}$ & $\begin{array}{l}\square \text { Increase of } \\
4-6 \text { stools per } \\
\text { day over } \\
\text { baseline; } \\
\text { moderate } \\
\text { increase in } \\
\text { ostomy output } \\
\text { compared to } \\
\text { baseline }\end{array}$ & $\begin{array}{l}\square \text { Increase of } \\
>=7 \text { stools per } \\
\text { day over } \\
\text { baseline; } \\
\text { incontinence; } \\
\text { hospitalization } \\
\text { indicated; } \\
\text { severe increase } \\
\text { in ostomy }\end{array}$ & \begin{tabular}{|l|}
\multicolumn{1}{|c|}{ Life- } \\
threatening \\
consequences; \\
urgent \\
intervention \\
indicated
\end{tabular} \\
\hline
\end{tabular}




\begin{tabular}{|c|c|c|c|c|c|}
\hline & & $\begin{array}{l}\text { compared to } \\
\text { baseline }\end{array}$ & & $\begin{array}{l}\text { output } \\
\text { compared to } \\
\text { baseline; } \\
\text { limiting self } \\
\text { care ADL }\end{array}$ & \\
\hline Nausea & $\square$ & $\begin{array}{l}\square \text { Loss of } \\
\text { appetite } \\
\text { without } \\
\text { alteration in } \\
\text { eating habits }\end{array}$ & \begin{tabular}{|l|} 
Oral intake \\
decreased \\
without \\
significant \\
weight loss, \\
dehydration or \\
malnutrition \\
\end{tabular} & $\begin{array}{l}\square \text { Inadequate } \\
\text { oral caloric or } \\
\text { fluid intake; } \\
\text { tube feeding; } \\
\text { TPN, or } \\
\text { hospitalization } \\
\text { indicated }\end{array}$ & \begin{tabular}{|l|}
$\square$ Life- \\
threatening \\
consequences; \\
urgent \\
intervention \\
indicated
\end{tabular} \\
\hline Vomiting & $\square$ & \begin{tabular}{|l}
\multicolumn{1}{|c}{$1-2$} \\
episodes \\
(separated \\
by 5 \\
minutes) in \\
24 hrs
\end{tabular} & \begin{tabular}{|l|} 
episodes \\
episodes \\
(separated by \\
5 minutes) in \\
$24 \mathrm{hrs}$ \\
\\
\end{tabular} & \begin{tabular}{|l}
$\square>=6$ \\
episodes \\
(separated by 5 \\
minutes) in 24 \\
hrs; tube \\
feeding; TPN \\
or \\
hospitalization \\
indicated
\end{tabular} & \\
\hline Fatigue & $\square$ & $\begin{array}{l}\square \text { Fatigue } \\
\text { relived by } \\
\text { rest }\end{array}$ & \begin{tabular}{|l|}
$\square$ Fatigue not \\
relieved by \\
rest; limiting \\
instrumental \\
ADL
\end{tabular} & $\begin{array}{l}\square \text { Fatigue not } \\
\text { relieved by } \\
\text { rest; limiting } \\
\text { self care ADL }\end{array}$ & \\
\hline \multicolumn{6}{|l|}{ Other: } \\
\hline Medications & \multicolumn{5}{|c|}{$\begin{array}{l}\text { Review current medications } \\
\text { Need for additional medications for symptom management } \\
\text { Prescription refills as appropriate }\end{array}$} \\
\hline \multicolumn{6}{|l|}{ "How are you coping?" } \\
\hline \multicolumn{6}{|l|}{$\begin{array}{l}\text { Review Phone Triage/ Sick } \\
\text { line information (including } \\
\text { when appropriate to call) }\end{array}$} \\
\hline $\begin{array}{l}\text { Next appointment } \\
\text { information: Are you able to } \\
\text { keep this appointment? If } \\
\text { not, can I help reschedule? }\end{array}$ & \multicolumn{5}{|c|}{ Next appointment: } \\
\hline Referrals & $\square \mathrm{MD}$ & $\square \mathrm{NI}$ & \begin{tabular}{l|l} 
P/PA & $\square$ \\
& Navigato \\
& RN
\end{tabular} & \begin{tabular}{l|l} 
or & $\square$ Social \\
Work
\end{tabular} & $\begin{array}{l}\square \text { Infusion } \\
\text { RN }\end{array}$ \\
\hline \multicolumn{6}{|l|}{ Comments/ Notes: } \\
\hline
\end{tabular}


Time spent on call

\section{Appendix B}

Education Session Outline Telephone Follow-Up Post Initial Chemotherapy

Objectives:

- Review development and implementation of standardization documentation/assessment tool

- Identify the driving force for telephone follow-up change

- Identify process for identification of appropriate patients and assignment of staff

- Identify and demonstrate competency in the elements of assessment in the post initial chemotherapy call guidelines

Background of quality improvement project

- $\quad$ Purpose of project

Review of current process

- Inconsistent telephone follow-up

- Inconsistent documentation with no template

Review of current literature

Review of post initial chemotherapy follow-up guidelines

Process for conducting call and documentation

- Call to be conducted within 48 hours to all first time chemotherapy patients 
- Documentation under telephone encounter within EHR with follow-up (MD/NP/PA) initiated for CTCAE score $>3$

- Support of management

- Utilization of charge nurse to allow for time for call backs

- Competency with documentation of call guidelines

Discussion of potential barriers 


\section{Appendix C}

\section{Post Evaluation Chemotherapy Follow-Up Script Guidelines}

Please circle the appropriate response in relation to your experience with the pilot of the chemotherapy follow-up script guidelines.

\section{The script guideline was easy to use}

1. Strongly Disagree 2. Disagree 3 . Neither agree nor disagree 4 . Agree 5. Strongly Agree

\section{The script guideline flowed logically}

1. Strongly Disagree $\quad$ 2. Disagree $\quad 3$. Neither agree nor disagree 4 . Agree 5. Strongly Agree

3. Assessment questions were appropriate to post chemotherapy patients

1. Strongly Disagree $\quad$ 2. Disagree $\quad 3$. Neither agree nor disagree 4 . Agree 5. Strongly Agree

4. Assessment questions elicited appropriate information from patient/family

1. Strongly Disagree 2. Disagree 3 . Neither agree nor disagree 4 . Agree 5. Strongly Agree

5. Nurse was able to document interventions performed i.e. referral to LIP, sick visit at clinic

1. Strongly Disagree 2. Disagree 3 . Neither agree nor disagree $\quad 4$. Agree 5. Strongly Agree 
6. Conducting call and completion of documentation took 30 minutes or less

1. Strongly Disagree 2. Disagree 3. Neither agree nor disagree 4. Agree 5. Strongly Agree

Additional Comments: 